\title{
Deterministic neural networks as sources of uncorrelated noise for probabilistic computations
}

\author{
Jakob Jordan ${ }^{1 *}$, Tom Tetzlaff ${ }^{1}$, Mihai Petrovici², Oliver Breitwieser ${ }^{2}$, Ilja Bytschok², Johannes Bill ${ }^{3}$, \\ Johannes Schemmel ${ }^{2}$, Karlheinz Meier², Markus Diesmann \\ From 24th Annual Computational Neuroscience Meeting: CNS*2015 \\ Prague, Czech Republic. 18-23 July 2015
}

Neural-network models of brain function often rely on the presence of noise [1-4]. To date, the interplay of microscopic noise sources and network function is only poorly understood. In computer simulations and in neuromorphic hardware [5-7], the number of noise sources (random-number generators) is limited. In consequence, neurons in large functional network models have to share noise sources and are therefore correlated. In general, it is unclear how shared-noise correlations affect the performance of functional network models. Further, there is so far no solution to the problem of how a limited number of noise sources can supply a large number of functional units with uncorrelated noise.

Here, we investigate the performance of neural Boltzmann machines [2-4]. We show that correlations in the background activity are detrimental to the sampling performance and that the deviations from the target distribution scale inversely with the number of noise sources. Further, we show that this problem can be overcome by replacing the finite ensemble of independent noise sources by a recurrent neural network with the same number of units. As shown recently, inhibitory feedback, abundant in biological neural networks, serves as a powerful decorrelation mechanism [8,9]: Shared-noise correlations are actively suppressed by the network dynamics. By exploiting this effect, the network performance is significantly improved. Hence, recurrent neural networks can serve as natural finite-size noise sources for functional neural networks, both in biological and in synthetic neuromorphic substrates. Finally we investigate the impact of sampling network parameters on its ability to faithfully represent a given well-

\footnotetext{
* Correspondence: j.jordan@fz-juelich.de

${ }^{1}$ Institute for Neuroscience and Medicine (INM-6) and Institute for Advanced Simulation (IAS-6), Jülich Research Center and JARA, Jülich, Germany Full list of author information is available at the end of the article
}

defined distribution. We show that sampling networks with sufficiently strong negative feedback can intrinsically suppress correlations in the background activity, and thereby improve their performance substantially.

\section{Acknowledgements}

Partially supported by the Helmholtz Association portfolio theme SMHB, the Jülich Aachen Research Alliance (JARA), EU Grant 269921 (BrainScaleS), The Austrian Science Fund FWF \#1753-N23 (PNEUMA), The Manfred Stärk Foundation, and EU Grant 604102 (Human Brain Project, HBP).

\section{Authors' details}

'Institute for Neuroscience and Medicine (INM-6) and Institute for Advanced Simulation (IAS-6), Jülich Research Center and JARA, Jülich, Germany. ${ }^{2}$ Kirchhoff Institute for Physics, Ruprecht-Karls-University Heidelberg, Heidelberg, Germany. Institute for Theoretical Computer Science, Graz University of Technology, Graz, Austria.

Published: 18 December 2015

\section{References}

1. Rolls ET, Deco G: The noisy brain. Oxford University Press; 2010.

2. Hinton GE, Sejnowski TJ, Ackley DH: Boltzmann machines: constraint satisfaction networks that learn. Technical report, Carnegie-Mellon University 1984.

3. Buesing L, Bill J, Nessler B, Maass W: Neural Dynamics as Sampling: A Model for Stochastic Computation in Recurrent Networks of Spiking Neurons. PlOS CB 2011, 7:e1002211.

4. Petrovici MA, Bill J, Bytschok I, Schemmel J, Meier K: Stochastic inference with deterministic spiking neurons 2013, arXiv 1311.3211v1 [q-bio.NC].

5. Schemmel J, Bruederle D, Gruebl A, Hock M, Meier K, Millner S: AWaferScale Neuromorphic Hardware System for Large-Scale Neural Modeling. Proceedings of the 2010 International Symposium on Circuits and Systems (ISCAS), IEEE Press 2010, 1947-1950.

6. Bruederle D, Petrovici M, Vogginger B, Ehrlich M, Pfeil T, Millner S, Gruebl A, Wendt K, Mueller E, Schwartz MO et al: A comprehensive workflow for general-purpose neural modeling with highly configurable neuromorphic hardware systems. Biological Cybernetics 2011, 104:263-296.

7. Petrovici MA, Vogginger B, Mueller $P$, Breitwieser $O$, Lundqvist $M$, Muller $L$, Ehrlich M, Destexhe A, Lansner A, Schueffny R, et al: Characterization and Compensation of Network-Level Anomalies in Mixed-Signal Neuromorphic Modeling Platforms. PLOS ONE 2014, 9(10):e108590. 
8. Renart A, De La Rocha J, Bartho P, Hollender L, Parga N, Reyes A, Harris KD: The asynchronous State in Cortical Circuits. Science 2010, 327:587-590.

9. Tetzlaff T, Helias M, Einevoll G, Diesmann M: Decorrelation of neuralnetwork activity by inhibitory feedback. PloS CB 2012, 8:e1002596.

doi:10.1186/1471-2202-16-S1-P62

Cite this article as: Jordan et al:: Deterministic neural networks as sources of uncorrelated noise for probabilistic computations. BMC Neuroscience 2015 16(Suppl 1):P62.

Submit your next manuscript to BioMed Central and take full advantage of:

- Convenient online submission

- Thorough peer review

- No space constraints or color figure charges

- Immediate publication on acceptance

- Inclusion in PubMed, CAS, Scopus and Google Scholar

- Research which is freely available for redistribution

Submit your manuscript at www.biomedcentral.com/submit
C Biomed Central 